\title{
Forelimb unloading impairs glenohumeral muscle development in growing rats
}

Sophia K. Tushak ${ }^{\mathrm{a}, 1}$, Margaret K. Tamburro ${ }^{\mathrm{a}, 1}$, Emily B. Fawcett ${ }^{\mathrm{a}}$, Lauren E. Merritt LE ${ }^{\mathrm{b}}$, Katherine R. Saul ${ }^{\text {, }}$ PhD, and Jacqueline H. Cole ${ }^{\mathrm{a}, *}, \mathrm{PhD}$

${ }^{1}$ These authors contributed equally to this work.

${ }^{a}$ Joint Department of Biomedical Engineering, University of North Carolina, Chapel Hill, NC, and North Carolina State University, Raleigh, NC, USA

${ }^{\mathrm{b}}$ Mechanical and Aerospace Engineering, North Carolina State University, Raleigh, NC, USA

*Corresponding author:

Dr. Jacqueline H. Cole, PhD

Assistant Professor

North Carolina State University \& University of North Carolina-Chapel Hill

4212B Engineering Building III

911 Oval Drive

Campus Box 7115

Raleigh, NC 27695-7115

Telephone: 919-515-5955

Email: jacquecole@,ncsu.edu 


\section{Abstract}

2 Proper joint loading is essential for healthy musculoskeletal development. Many pediatric

3 neuromuscular disorders cause irreversible muscle impairments resulting from both physiological

4 changes and mechanical unloading of the joint. While previous studies have examined the effects

5 of hindlimb unloading on musculoskeletal development in the lower limb, none have examined

6 solely forelimb unloading. Thus, a large deficit in knowledge of the effect of upper limb unloading

7 exists and must be addressed in order to better understand how the glenohumeral joint adapts

8 during development. Two forelimb unloading models were developed to study the effects of

9 varying degrees of unloading on the glenohumeral joint in growing rats: forelimb suspension $(\mathrm{n}=6$, intervention 21 days post-natal) with complete unloading of both limbs via a novel suspension

11 system and forearm amputation ( $\mathrm{n}=8$, intervention 3-6 days post-natal) with decreased loading and

12 limb use in one limb after below-elbow amputation. After 8 weeks of unloading, changes in muscle

13 architecture and composition were examined in ten muscles surrounding the shoulder. Results

14 were compared to control rats from a previous study $(\mathrm{n}=8)$. Both methods of altered loading

15 significantly affected muscle mass, sarcomere length, and optimal muscle length compared to 16 control rats, with the biceps long head and triceps long head observing the most marked

17 differences. Forearm amputation also significantly affected muscle mass, sarcomere length, and 18 optimal muscle length in the affected limb relative to the contralateral limb. Muscle composition, 19 assessed by collagen content, remained unchanged in all groups. This study demonstrated that 20 forearm amputation, which was administered closer to birth, had greater effects on muscle than 21 forelimb suspension, which was administered a few weeks later than amputation. 


\section{Introduction}

Mechanical loading is critically important for healthy musculoskeletal development ${ }^{1,2}$ and maintenance $\mathrm{e}^{3,4}$. In adult murine models, unloading via hindlimb suspension, microgravity during spaceflight, and muscle paralysis causes changes in muscle architecture. For example, unloading in adult murine animals caused substantial reductions of $41-66 \%$ in skeletal muscle size, mass, and strength $^{6,8,9}$, as well as up to $13 \%$ longer sarcomere lengths ${ }^{8}$. However, muscle composition measured by collagen content was unaffected by unloading in adult rodents ${ }^{7,11}$. In growing animals, unloading is particularly impactful, causing irreversible musculoskeletal changes ${ }^{5,12,56}$, including altered joint morphology ${ }^{12}$, which influences surrounding muscle, and decreased muscle mass by over 3 to 5 -fold ${ }^{5,56}$. However, the effects of unloading on other muscle architecture metrics (e.g., sarcomere length, optimal muscle length) and muscle composition (e.g., collagen content) have not previously been examined in growing animals.

Unloading models have traditionally focused on the hindlimbs ${ }^{15}$, resulting in limited understanding of the specific contributions of forelimb unloading to changes in muscle of glenohumeral joint, particularly during development. With a combined incidence of more than 5 per 1,000 live births ${ }^{16-19}$, pathologies affecting the developing muscles surrounding the glenohumeral joint (e.g., brachial plexus birth injury ${ }^{20-22}$, congenital muscular dystrophy ${ }^{23-25}$, cerebral palsy ${ }^{26-28}$, and congenital myasthenia gravis ${ }^{29-31}$ ) have substantial implications for the effects of altered forelimb loading during development. However, isolating the role that altered loading plays in these conditions is challenging, since, for example, nerve injury also directly contributes to detrimental muscle ${ }^{12,13}$ changes. Hence, the independent contributions of altered mechanical loading and nerve injury or other congenital changes to musculoskeletal development following neuromuscular disorders and injuries are difficult to elucidate. Understanding the contributions of unloading to these pathologies is an important step in determining which changes 
47 result directly from injury or disease and which are a functional consequence of altered joint

48 loading, which may aid treatment development to restore muscle function.

Because of anatomical similarities to human shoulders ${ }^{32,33}$ and rapid development to

50 skeletal maturity ${ }^{34}$, murine models are often used to study these injuries and diseases ${ }^{20-31}$. While

51 murine hindlimb unloading ${ }^{35}$ and partial unloading paradigms exist ${ }^{36}$, to date no study has

52 implemented a partial or complete forelimb unloading paradigm that targets the glenohumeral joint

53 during neonatal development. A previous mouse model of bipedal locomotion training to improve

54 function following spinal cord injury involved forelimb unloading, although these effects were not

55 evaluated, and the animal was placed in an unnaturally upright posture, making this model

56 unsuitable for studying long-term forelimb unloading ${ }^{37}$. We implemented two novel murine

57 models to simulate variable degrees of mechanical unloading in the glenohumeral joint that occurs

58 with this array of neuromuscular diseases and injuries: forelimb suspension and forearm

59 amputation. Our objective was to determine the effect of these forelimb unloading models on the

60 postnatal development of muscles surrounding the glenohumeral joint, in particular muscle

61 architecture and composition.

62

Methods

All procedures were approved by the Institutional Animal Care and Use Committee at

North Carolina State University prior to the start of the study. Male and female Sprague Dawley rats (Charles River Laboratories, Wilmington, MA) were subjected to forelimb unloading using one of two different methods (Fig. 1): forelimb suspension $(n=6)$ or forearm amputation $(n=8)$. Results from the unloading groups were compared to results from a control group $(n=8)$. 


\section{Forelimb Suspension}

Six Sprague Dawley rats (2 female, 4 male) from three litters were exposed to forelimb unloading promptly after weaning at 3 weeks of age. Rats were placed in fitted harnesses, connected to a custom suspension system, and subjected to a six-week period of continuous unloading in both forelimbs (Fig. 2). Details of our suspension system were described previously ${ }^{38}$. The rats experienced a 12-hour light/12-hour dark cycle. Rat chow (Purina, Woodstock, Ontario, Canada) and HydroGel ${ }^{\circledR}\left(\right.$ ClearH2O ${ }^{\circledR}$, Inc., Westbrook, ME) were offered ad libitum. HydroGel ${ }^{\circledR}$ was used instead of water, because the typical water bottle interfered with the suspension system. At 9 weeks of age and after six weeks of loading, the rats were euthanized with $\mathrm{CO}_{2}$ inhalation followed by bilateral thoracotomy.

\section{Forearm Amputation}

Eight Sprague Dawley rat pups (2 female, 6 male) from the same three litters as the forelimb suspension group received forearm amputations at 3-6 days of age. Rat pups were anesthetized with isoflurane, and right forearms were amputated through elbow disarticulation using aseptic technique, with the contralateral forelimbs remaining intact. The wound was irrigated and closed with tissue adhesive and suture. To minimize pain, rats received a local anesthetic (bupivacaine) at the incision site during surgery, one dose of buprenorphine $(0.01 \mathrm{mg} / \mathrm{kg})$ and carprofen $(5 \mathrm{mg} / \mathrm{kg})$ immediately after surgery, and a course of carprofen once per day for five days after surgery. Upon recovery from anesthesia, rat pups were returned to their dams and regularly monitored for signs of acceptance. Rat pups were weaned from their dams at 3 weeks of age and housed three per cage in the same room with the same accommodations as the forelimb 
92 suspension group, except they were given a typical water bottle. At 8 weeks of age, the rats were

93 euthanized with $\mathrm{CO}_{2}$ inhalation followed by bilateral thoracotomy.

95 Control

Control comparison data were obtained from previously assessed rats that underwent a

97 sham surgery ${ }^{50}$. In that study, 8 Sprague Dawley rat pups ( 3 female, 5 male) from three litters received sham surgeries at 3-6 days of age that exposed the brachial plexus nerve bundle through the right pectoralis major, but no subsequent nerve injury was administered, and the contralateral forelimbs were kept intact. The wound was irrigated and closed with tissue adhesive. To minimize pain, one dose each of buprenorphine and carprofen was administered immediately following surgery. Rats received the same post-surgical care as the forearm amputation group. At 8 weeks of age, the rats were euthanized with $\mathrm{CO}_{2}$ inhalation followed by bilateral thoracotomy. For the control group, the left forelimb - which did not undergo surgery - was considered unaffected and used for comparison to the unloading groups.

\section{Muscle Dissection}

Following euthanasia, the upper body was harvested using a guillotine to remove both the head and lower body. The torso was then fixed in 10\% neutral buffered formalin for two days and stored in $70 \%$ ethanol at $4^{\circ} \mathrm{C}$ until muscle dissection. In 11 rats (5 control, 3 suspension, 3

111 amputation), 10 muscles surrounding the shoulder and upper forelimb were dissected bilaterally

112 and stored in $70 \%$ ethanol at $4^{\circ} \mathrm{C}$ until architecture analysis: pectoralis major, acromiodeltoid, 113 spinodeltoid, biceps long head, biceps short head, subscapularis, supraspinatus, infraspinatus, teres 114 major, and triceps long head ${ }^{39}$. In the remaining 11 rats (3 control, 3 suspension, 5 amputation), 
115 four muscles (biceps long head, biceps short head, upper and lower subscapularis) were harvested

116 bilaterally for composition analysis. The proximal end of each muscle was embedded in optimum

117 cutting temperature compound and set in 2-methylbutane cooled by liquid nitrogen, and the entire

118 muscle was then snap frozen and stored at $-80^{\circ} \mathrm{C}$ until sectioning.

122 excess ethanol, muscles were weighed on a digital scale (resolution of $0.01 \mathrm{~g}$ ). For each muscle, 9

123 muscle fibers were extracted, 3 each from the proximal, middle, and distal regions of the muscle.

124 Sarcomere lengths were measured via a 5.0-mW HeNe laser with a wavelength of $633 \mathrm{~nm}$

125 (Thorlabs, Newton, NJ) using an established laser diffraction method ${ }^{26}$. All muscle lengths and

126 distances between each diffraction band were measured using digital calipers (resolution of 0.01

$127 \mathrm{~mm}$ ). The 9 sarcomere measurements were averaged to find the mean sarcomere length for each

128 muscle. To determine the excursion capacity of the muscles and account for possible stretch in the

129 fixed muscle as indicated by sarcomere length, optimal muscle length was calculated ${ }^{40}$ :

$$
L_{0}^{m}=L^{m}\left(\frac{2.4 \mu m}{L^{s}}\right)
$$

where $L^{m}$ is muscle length and $L^{S}$ is sarcomere length. The optimal sarcomere length corresponded to that of rat skeletal muscle $(2.4 \mu \mathrm{m})$.

\section{Muscle Fibrosis}

In muscles stored at $-80^{\circ} \mathrm{C}$, three transverse cryosections with a thickness of $10 \mu \mathrm{m}$ were

136 obtained from each muscle (Cryotome FSE Cryostat, Thermo Scientific, Halethorpe, MD), 137 mounted to a silanized slide, and stored at $-80^{\circ} \mathrm{C}$ prior to staining. Muscle sections were stained 
with Masson's trichrome (American MasterTech, Lodi, CA) to identify collagen I deposition, a measure of fibrosis and muscle stiffening, and imaged at 20X magnification with light microscopy $\left(\right.$ EVOS $^{\circledR}$ FL Cell Imaging System, Thermo Scientific, Halethorpe, MD) In three sections per muscle, collagen content was calculated as the ratio of collagen area to muscle tissue area using a custom image processing protocol (MATLAB ${ }^{\circledR}$, The MathWorks, Inc., Natick, MA).

\section{Statistical Analyses}

To verify whether side-to-side differences were insignificant for the forelimb suspension and control groups (as expected) and to identify whether differences existed in muscle metrics between the affected and unaffected forelimbs for the amputation group, paired t-tests were used. Muscle architecture (mass, sarcomere length, optimal muscle length) and composition (collagen content) metrics were compared across the three groups (control, forelimb suspension, forearm amputation) using one-way ANOVA with Tukey's post-hoc tests. For the group comparisons, data from only one forelimb was used: right for both unloading groups and left (unoperated) for the control group. All analyses were performed in RStudio Cloud (v. alpha, The R Foundation for Statistical Computing, Vienna, Austria) with a significance level of $\alpha=0.05$.

\section{Results}

Side Differences Within Each Group

In the control and forelimb suspension groups, no significant side-to-side differences were found for any of the metrics, apart from the pectoralis major muscle mass in the control group, which was lower in the sham limb (right) compared to the unimpaired limb (left) as previously reported $^{39}$. This was expected, because the sham surgery involved a transverse infraclavicular 
161 incision through the pectoralis major to expose the brachial plexus. In the forearm amputation

162 group, muscle mass was an average of $37.7 \%$ lower for muscles in the right (affected) limb

163 compared to the left (unaffected) limb (Table 1). Affected limb muscle mass was significantly

164 lower than unaffected for acromiodeltoid $(18.1 \pm 1.7 \%, \mathrm{p}=0.0153)$, biceps long head $(54.9 \pm$

$1657.9 \%, \mathrm{p}=0.0136)$, and triceps long head $(56.8 \pm 6.0 \%, \mathrm{p}=0.0136)$.

In the forearm amputation group, sarcomere length was not different between limbs for

167 most muscles. Sarcomeres were significantly longer in the affected biceps long head $(17.6 \pm 1.3 \%$,

$168 \mathrm{p}=0.0005)$ compared to unaffected muscles (Table 1). However, on average optimal muscle

169 lengths were an average of $22.7 \%$ shorter in muscles of the affected limb compared to the

170 unaffected limb. Optimal muscle lengths were significantly shorter in affected acromiodeltoid

$171(27.1 \pm 3.9 \%, \mathrm{p}=0.010)$ and biceps long head $(39.6 \pm 3.1 \%, \mathrm{p}=0.0002)$ compared to the

172 unaffected side.

173 Collagen content, indicative of muscle fibrosis, did not differ significantly between left and 174 right limbs in any group.

175

\section{Group Differences}

Muscle mass differed significantly across groups for biceps long head and triceps long head

178 (Fig. 3, Table 1). Compared to the control group, the forearm amputation group had significantly

179 lower average muscle mass in the biceps long head $(51.0 \%, p=0.0202)$ and triceps long head

$180(57.7 \%, \mathrm{p}=0.0229)$. Similarly, compared to the suspension group, the amputation group had lower 181 average muscle mass in the biceps long head $(51.6 \%, \mathrm{p}=0.0202)$ and triceps long head $(56.9 \%, \mathrm{p}$

$182=0.0437)$. No significant differences in muscle mass were found between the forelimb suspension 183 and control groups. 
Group differences were also observed in sarcomere length for acromiodeltoid,

185

186

187

188

189

190

191

192

193

194

195

196

197

198

199

200

201

202

203

204

205

206 spinodeltoid, biceps long head, subscapularis, supraspinatus, and teres major (Fig. 4, Table 1).

Compared to the control group, the amputation group had, on average, significantly shorter sarcomeres in the acromiodeltoid $(10.9 \%, \mathrm{p}=0.0235)$ and subscapularis $(10.4 \%, \mathrm{p}=0.0377)$ muscles but longer sarcomeres in spinodeltoid $(12.6 \%, \mathrm{p}=0.000612)$ and teres major $(25.7 \%, \mathrm{p}=$ 0.0000366). Compared to those in the suspension group, average sarcomere length in the amputation group was significantly longer in biceps long head $(17.4 \%, p=0.000300)$ and teres major $(9.2 \%, \mathrm{p}=0.00212)$. Compared to control, the suspension group had significantly shorter sarcomeres in the biceps long head $(8.5 \%, \mathrm{p}=0.0449)$, subscapularis $(13.5 \%, \mathrm{p}=0.0102)$, and supraspinatus $(14.6 \%, \mathrm{p}=0.0401)$ muscles but longer sarcomeres in spinodeltoid $(8.3 \%, \mathrm{p}=$ $0.00792)$ and teres major $(15.1 \%, \mathrm{p}=0.00148)$.

Optimal muscle length differed by group for biceps long head, biceps short head, and triceps long head (Fig. 5, Table 1). Compared to control, average optimal muscle length in the amputation group was significantly shorter for biceps long head $(30.1 \%, p=0.0145)$ and triceps long head $(28.1 \%, \mathrm{p}=0.00185)$, indicating reduced longitudinal growth. Similarly, compared to suspension, average optimal muscle lengths in the amputation group were significantly shorter for biceps long head $(36.9 \%, p=0.00493)$, biceps short head $(27.0 \%, p=0.0273)$, and triceps long head $(35.8 \%, p=0.000372)$.

Qualitative analysis of histologic images revealed minimal differences across the groups in collagen staining for the 4 analyzed muscles (biceps long head, biceps short head, upper and lower subscapularis muscles) (Fig. 6). Quantitative analysis of these images showed that the ratio of collagen area to total muscle area did not differ significantly across the three groups for any of the 4 muscles examined (Table 2). 


\section{Discussion}

Unloading with the two models had different effects on the growth of forelimb muscles.

210 The suspension group did not affect muscle mass oroptimal muscle lengths relative to control,

211 except for an increased optimal length for biceps short head. In contrast, the amputation

212 intervention led to lower muscle mass and optimal muscle length for several muscles in the

213 affected forelimb compared to the contralateral limb, suggesting that unloading via forearm

214 amputation during postnatal development can inhibit muscle growth. Specifically, muscle mass

215 was lower in the acromiodeltoid, biceps long head, and triceps long head, and optimal muscle

216 length was shorter in the acromiodeltoid and biceps long head following amputation. The

217 amputated group also had lower muscle mass and shorter optimal muscle length in the affected

218 limb compared to the forelimb suspension (right affected limb) and control (unaffected limb)

219 groups. On average, the amputated biceps and triceps long head muscles were approximately half

220 the mass and $75 \%$ of the optimal length of the corresponding muscles in the suspended and control

221 groups.

The amputation procedure provides an explanation for the specific affected muscles in this

223 group. The biceps long head, biceps short head, and triceps long head originate at the scapula and

224 insert to the proximal radius or ulna. During the amputation procedure, the severing at the insertion

225 point releases the muscles and causes widespread denervation and atrophy, leading to reduced

226 muscle mass ${ }^{52}$. In other studies found that denervated extensor digitorum longus muscle mass in

227 growing rats increased after initial atrophy, following similar growth patterns as the control

228 contralateral limbs, but soleus muscle mass decreased relative to the control ${ }^{53}$. The authors

229 suggested that the increased growth was due to elevated protein synthesis after continued 
230 lengthening of the muscle, while the decrease in growth was attributed to a reduction in protein

231 synthesis after continued shortening of the muscle. Although the biceps short head was denervated,

232 it likely experienced extended periods of lengthening, causing it to grow similar to the suspension

233 and control groups. The biceps and triceps likely experienced shortening over the duration of the

234 study due to the release at amputation, which contributed to muscle mass loss and shortening. The

235 other forelimb muscles were not affected by the amputation procedure and therefore there was no

236 marked differences in muscle architecture.

The suspension group also exhibited changes in muscle architecture relative to the control

238 group, which may be explained by the relative immobilization of the limbs. For example,

239 immobilization in innervated lower limb muscles in growing rats found that a decrease in muscle

240 mass compared to a control was attributed to higher levels of protein breakdown and reduced

241 protein synthesis in the affected muscles when the muscles were held in a shortened position ${ }^{54}$.

242 When held in a lengthened position, immobilized muscles in the lower limbs of growing rats

243 exhibited slightly increased muscle mass compared to the control, which was attributed to

244 decreased protein breakdown and increased protein synthesis during active and passive activity of

245 the muscles $^{54}$. The rats in the suspension group, although immobilized in the upper limbs,

246 experienced typical muscle activity as seen in control rats while performing daily eating and

247 cleaning activities, so muscle mass was not significantly affected by limb unloading because rats

248 had full mobility of the unloaded limbs.

Altered loading had a broader and more varied impact on sarcomere length. Within the

250 amputation group, the biceps long head had shortened optimal length but longer sarcomere length

251 on the affected side compared to the unaffected side. The suspension and amputation groups

252 exhibited similar changes in muscle sarcomere length across the different muscles, with shorter 
sarcomeres in subscapularis, and longer sarcomeres in spinodeltoid and teres major, compared to control, with additional varied effects in the acromiodeltoid and biceps long head. Hindlimb unloading has been shown to reduce titin density in the adult female rat soleus and plantaris muscles $^{55}$. Since titin plays an integral part in sarcomere positional stability, significant losses in titin composition causes vast changes in contractile activity and likely force production. Sarcomere length contributes to optimal muscle length and muscle force production. Any deviation from the optimal sarcomere length causes actin and myosin to inefficiently interact, which limits force production in the muscle. Force production in a muscle-tendon unit, however, is not only governed by muscle length; cross-sectional area is directly proportional to the amount of force each muscle can harvest ${ }^{49}$ and is likely affected by changes in muscle mass ${ }^{51}$. Although sarcomere lengths for the anteriodeltoid, spinodeltoid, subscapularis, supraspinatus, and teres major were markedly different in the unloading groups, optimal muscle lengths for these muscles remained the same compared to the control group. Since optimal muscle length is a ratio of sarcomere length to measured muscle length, the unchanged optimal muscle length across groups is likely due to similar changes in sarcomere and optimal muscle lengths. Based on this, the biceps long head and triceps long head, which displayed remarkably lower muscle mass and longer sarcomere lengths that translated to shorter optimal muscle lengths, experienced the greatest decrease in muscletendon force production across the board.

These results are consistent with a previous study that investigated changes in muscle architecture in growing rats after neonatal injury to the brachial plexus nerve $\mathrm{e}^{51}$. When comparing the affected limb to the contralateral limb, muscle mass in the same ten muscles as in this study was significantly less in all but one observed muscle, including the biceps long head and triceps long head, similar to the amputation group in this study. Concurrent to the previous study, 
sarcomeres in the amputation group were significantly longer in the teres major and biceps long

277 head, along with the teres major in the suspension group relative to the control group. Unlike the

278 injury groups, the suspension group, however, did exhibit shorter sarcomeres in the biceps long

279 head compared to the control group. This comparison shows that muscle mass and sarcomere

280 length in the amputation group more closely mimicked those of injury groups seen in the literature.

281 Optimal muscle length was shorter in the biceps long head for both unloading groups, and biceps

282 short head for the suspension group, which showed that the suspension group more closely

283 resembled the injury groups seen in literature. The triceps long head was significantly affected by

284 both unloading methods, but not by nerve injury, which could mean that the triceps long head

285 muscle length is more sensitive to changes in loading than denervation.

Altered loading did not have a significant impact on muscle collagen content, with similar

groups. These findings are consistent with previous studies in adult female rat soleus muscle after

2 weeks of hindlimb unloading via tail-casting ${ }^{11}$. Although muscle fibrosis has been observed in children with neuromuscular disorders like cerebral palsy ${ }^{42}$ and with nerve injury ${ }^{43,51}$, our results indicate that fibrosis is unlikely to result as a consequence of reduced loading, and may instead result from other factors such as direct tissue injury or other physiological consequence..

295 found that mass in three leg muscles were not significantly affected by 30 days in space where 296 observed grooming rate was high ${ }^{9}$. Since the mice maintained daily grooming activity, the muscles 297 were activated throughout unloading, and these results are similar to our forelimb unloading condition with limited muscle effects. Previous hindlimb unloading studies reported muscle 
299

300

301

302

303

304

305

306

307

308

309

310

311

312

313

314

315

316

317

318

319

320

321

atrophy and decreased muscle mass One study examined the effect of 30-day space flight on 19week old mice and found that hindlimb muscle mass was not significantly affected by weightlessness but trended towards decreased soleus and extensor digitorum longus mass in the unloading groups ${ }^{9}$. This could be close to the cut-off of growing and adult. Another study using a tail-casting hindlimb unloading model in young adult female rats found that the addition of combined isometric, concentric, and eccentric muscle stimulation dampened muscle mass loss compared to the untrained contralateral limb, and muscle mass was unchanged from the regular weight-bearing group ${ }^{44}$. This could help adult rats maintain their muscle mass during unloading. In a partial weight-bearing study, 10-week old adult female mice gastrocnemius muscle was found to be significantly lower mass than that of the control groups ${ }^{36}$. Another hindlimb unloading study with adult male rats found that soleus, plantaris, adductor longus, gastrocnemius, and tibialis anterior muscle mass was significantly reduced after hindlimb unloading compared to typical weight-bearing. Isometric exercise attenuated the effects of unloading in the soleus by $54 \%$. Isometric exercise, however, did not aid the gastrocnemius and plantaris in maintaining muscle mass, as they were significantly less than control by $15 \%{ }^{45}$. Hindlimb unweighting was further determined as a cause for reduced muscle mass in adult rats ${ }^{47}$. Soleus muscle mass was significantly reduced in hindlimb unloaded growing rats compared to control rats after 17 days of unloading ${ }^{46}$. This effect was reversed after a 28 -day reambulation period. The authors noted that during hindlimb unloading, the ankle was plantarflexed, which caused shortening of the soleus and reduced muscle mass ${ }^{54}$. The mechanism of unloading largely affects muscle properties. If the limb is held in place by a cast, it could be immobilized in a shortened position, which has been shown to have detrimental effects on muscle. In a model in which the unloaded limbs are exposed, they can be held at a natural, optimal position, which may not have as much of an effect of muscle. 
Our forelimb suspension system differs from hindlimb suspension systems in that, although

324 loading and muscle activity during daily grooming and eating activities. Because muscle mass and

325 optimal muscle length were, for the most part, similar between the suspension and control groups,

326 this small amount of loading seems sufficient to stimulate normal forelimb muscle growth.

327 However, our forearm amputation group experienced both reduced weight bearing and reduced

328 limb use following amputation and were unable to walk on or groom with the amputated limb

329 normally. The affected limb served only as an occasional weight-bearing stabilizer, and forelimb

330 muscle use during these daily activities was greatly reduced. Therefore, the reduced muscle mass

331 observed in this group, compared both to the contralateral limb and to the suspension and control

332 groups, may result from limb disuse rather than direct unloading of the muscles.

\section{Limitations}

Both male and female rats were compared together, and sex differences were not considered. Young (3-month old) male rats have shown to display greater reduction in total body

337 mass compared to control rats over the hindlimb unloading period, while females did not ${ }^{48}$. The

338 amputation group comprised of 3 male rats, whereas the other groups had at least one female,

339 which could help explain why the amputation group displayed greater significance. The forelimb

340 suspension group was sacrificed one week later than the forelimb amputation and control groups

341 to accommodate the four male rats that were removed from the suspension system for a brief 4-

342 day period due to elevated stress, as indicated by lesions underneath the harness and porphyrin

343 discharge around the nose and eyes ${ }^{36}$. The removal occurred during the third week of unloading,

344 but the rats progressed normally after the wounds healed. In the future, an additional layer of 
345 breathable fabric should be placed between the harness and rat to reduce the amount of chaffing 346 and discomfort over the long unloading period. The suspension system, while removing weight

347 bearing from the forelimbs, did not completely eliminate loading, as the animals were able to

348 continue normal grooming and feeding activities, as noted above. With forearm amputation,

349 because the affected limb experienced reduced weight bearing and overall use, the contralateral

350 limb likely was loaded more throughout the study, potentially augmenting the side differences

351 observed. Nevertheless, similar muscle changes were observed for the affected limb compared to

352 the normally loaded limb of the control group.

\section{Conclusions}

355 Altered loading affected upper forelimb muscle mass and optimal muscle length, primarily

356 in the biceps and triceps muscles of the forearm amputation group. The forelimb suspension group

357 did not experience marked differences from the control group, showing that this unloading 358 paradigm did not negatively impact muscle growth and function, as in the forearm amputation

359 group. Our results suggest that even limited amounts of forelimb loading during non-weight360 bearing activities offset the unloading detriments observed in hindlimb unloading models, and 361 general limb use is more important for muscle growth than weight bearing. The muscle responses 362 in the amputation group more closely mimicked those results seen in nerve injury groups, making 363 this a more suitable model to assess isolated muscle effects due to forelimb unloading. 


\section{References}

365 1. Arvind V, Huang AH. 2017. Mechanobiology of limb musculoskeletal development. Ann N Y Acad Sci 1409(1):18-32.

367

368

369

370

371

372

373

374

375

376

377

378

379

380

2. Felsenthal N, Zelzer E. 2017. Mechanical regulation of musculoskeletal system development. Development 144(23):4271-4283.

3. Qin Y-X, Hu M. 2014. Mechanotransduction in Musculoskeletal Tissue Regeneration: Effects of Fluid Flow, Loading, and Cellular-Molecular Pathways. BioMed Research International [cited 2019 Feb 20] Available from: https:/www.hindawi.com/journals/bmri/2014/863421/.

4. Shwartz Y, Blitz E, Zelzer E. 2013. One load to rule them all: Mechanical control of the musculoskeletal system in development and aging. Differentiation 86(3):104-111.

5. Klein L, Heiple KG, Stromberg BV. 1983. Comparison of growth-induced resorption and denervation-induced resorption on the release of $[3 \mathrm{H}]$ tetracycline, 45 calcium, and $[3 \mathrm{H}]$ collagen from whole bones of growing rats. J. Orthop. Res. 1(1):50-56.

6. Laib A, Barou O, Vico L, et al. 2000. 3D micro-computed tomography of trabecular and cortical bone architecture with application to a rat model of immobilisation osteoporosis. Med. Biol. Eng. Comput. 38(3):326-332.

7. Warner SE, Sanford DA, Becker BA, et al. 2006. Botox induced muscle paralysis rapidly degrades bone. Bone 38(2):257-264.

8. Bouvard B, Mabilleau G, Legrand E, et al. 2012. Micro and macroarchitectural changes at the tibia after botulinum toxin injection in the growing rat. Bone 50(4):858-864.

9. Tascher G, Brioche T, Maes P, et al. 2017. Proteome-wide Adaptations of Mouse Skeletal Muscles during a Full Month in Space. J. Proteome Res. 16(7):2623-2638. 
10. Yu Q, Morales M, Li N, et al. 2018. Skeletal, cardiac, and respiratory muscle function and histopathology in the P448Lneo- mouse model of FKRP-deficient muscular dystrophy. Skelet Muscle 8 [cited 2018 Nov 7] Available from: https:/www.ncbi.nlm.nih.gov/pmc/articles/PMC5889611/.

11. Heinemeier KM, Olesen JL, Haddad F, et al. 2009. Effect of unloading followed by reloading on expression of collagen and related growth factors in rat tendon and muscle. J. Appl. Physiol. 106(1):178-186.

12. Ohira Y, Kawano F, Wang XD, et al. 2006. Irreversible morphological changes in leg bone following chronic gravitational unloading of growing rats. Life Sciences 79(7):686-694.

13. Globus RK, Morey-Holton E. 2016. Hindlimb unloading: rodent analog for microgravity. Journal of Applied Physiology 120(10):1196-1206.

14. Abicht A, Müller J, Lochmüller H. 1993. Congenital Myasthenic Syndromes. In: Adam MP, Ardinger HH, Pagon RA, et al., editors. GeneReviews ${ }^{\circledR}$. Seattle (WA): University of Washington, Seattle [cited 2018 Nov 8] Available from: http://www.ncbi.nlm.nih.gov/books/NBK1168/.

15. Sparks SE, Quijano-Roy S, Harper A, et al. 1993. Congenital Muscular Dystrophy Overview. In: Adam MP, Ardinger HH, Pagon RA, et al., editors. GeneReviews ${ }^{\circledR}$. Seattle (WA): University of Washington, Seattle [cited 2018 Nov 8] Available from: http://www.ncbi.nlm.nih.gov/books/NBK1291/.

16. Stavsky M, Mor O, Mastrolia SA, et al. 2017. Cerebral Palsy-Trends in Epidemiology and Recent Development in Prenatal Mechanisms of Disease, Treatment, and Prevention. Front $\left.\begin{array}{lllll}\text { Pediatr } & 5 & \text { [cited } & 2018 \text { Nov }\end{array}\right]$ Available from: https://www.ncbi.nlm.nih.gov/pmc/articles/PMC5304407/. 
17. Chauhan SP, Blackwell SB, Ananth CV. 2014. Neonatal brachial plexus palsy: incidence, prevalence, and temporal trends. Semin. Perinatol. 38(4):210-218.

412 18. Soldado F, Fontecha CG, Marotta M, et al. 2014. The role of muscle imbalance in the pathogenesis of shoulder contracture after neonatal brachial plexus palsy: a study in a rat model. J Shoulder Elbow Surg 23(7):1003-1009.

19. Wang B, Chen L, Liu B, et al. 2012. Differentiation of endogenous neural stem cells in adult versus neonatal rats after brachial plexus root avulsion injury. Neural Regen Res 7(23):17861790.

20. Wang Z-Q, Xiu D-H, Liu G-F, Jiang J-L. 2018. Overexpression of Neuregulin-1 (NRG-1) Gene Contributes to Surgical Repair of Brachial Plexus Injury After Contralateral C7 Nerve Root Transfer in Rats. Med. Sci. Monit. 24:5779-5787.

421 21. Rodrigues M, Echigoya Y, Fukada S, Yokota T. [date unknown]. Current Translational 422 Research and Murine Models For Duchenne Muscular Dystrophy. J Neuromuscul Dis 3(1):29-48.

424 22. Dowling JJ, D. Gonorazky H, Cohn RD, Campbell C. 2018. Treating pediatric neuromuscular 425 disorders: The future is now. Am J Med Genet A 176(4):804-841.

426

23. Clowry GJ, Basuodan R, Chan F. 2014. What are the Best Animal Models for Testing Early Intervention in Cerebral Palsy? Front Neurol 5 [cited 2018 Nov 8] Available from: https://www.ncbi.nlm.nih.gov/pmc/articles/PMC4255621/. Model of Cerebral Palsy. J Vis Exp (117) [cited 2018 Nov 8] Available from: https:/www.ncbi.nlm.nih.gov/pmc/articles/PMC5226120/. 
432

433

434

435

436

437

438

439

440

441

442

443

444

445

446

447

448

449

450

451

452

453

454
25. Tan J, Zheng X, Zhang S, et al. 2014. Response of the sensorimotor cortex of cerebral palsy rats receiving transplantation of vascular endothelial growth factor 165-transfected neural stem cells. Neural Regen Res 9(19):1763-1769.

26. Ban J, Phillips WD. 2015. Mouse models of myasthenia gravis. Curr. Pharm. Des. 21(18):2468-2486.

27. [Date unknown]. Fatigue and Muscle Atrophy in a Mouse Model of Myasthenia Gravis Is Paralleled by Loss of Sarcolemmal nNOS. [cited 2018 Nov 8] Available from: https://journals.plos.org/plosone/article?id=10.1371/journal.pone.0044148.

28. Mantegazza R, Cordiglieri C, Consonni A, Baggi F. 2016. Animal models of myasthenia gravis: utility and limitations. Int J Gen Med 9:53-64.

29. Lee S, Shin J, Hong Y, et al. 2012. Beneficial effects of melatonin on stroke-induced muscle atrophy in focal cerebral ischemic rats. Lab Anim Res 28(1):47-54.

30. Biering-Sørensen B, Kristensen IB, Kjaer M, Biering-Sørensen F. 2009. Muscle after spinal cord injury. Muscle Nerve 40(4):499-519.

31. Borschmann KN, Rewell SS, Iuliano S, et al. 2017. Reduced bone formation markers, and altered trabecular and cortical bone mineral densities of non-paretic femurs observed in rats with ischemic stroke: A randomized controlled pilot study. PLoS ONE 12(3):e0172889.

32. Norlin R, Hoe-Hansen C, Oquist G, Hildebrand C. 1994. Shoulder region of the rat: anatomy and fiber composition of some suprascapular nerve branches. Anat. Rec. 239(3):332-342.

33. Soslowsky LJ, Carpenter JE, DeBano CM, et al. 1996. Development and use of an animal model for investigations on rotator cuff disease. J Shoulder Elbow Surg 5(5):383-392.

34. Quinn R. 2005. Comparing rat's to human's age: how old is my rat in people years? Nutrition 21(6):775-777. 
35. Morey-Holton ER, Globus RK. 2002. Hindlimb unloading rodent model: technical aspects. J. Appl. Physiol. 92(4):1367-1377.

36. Wagner EB, Granzella NP, Saito H, et al. 2010. Partial weight suspension: a novel murine model for investigating adaptation to reduced musculoskeletal loading. J. Appl. Physiol. 109(2):350-357.

37. van den Brand R, Heutschi J, Barraud Q, et al. 2012. Restoring voluntary control of locomotion after paralyzing spinal cord injury. Science 336(6085):1182-1185.

38. Tushak S, Tamburro M. 2018. Development of a Rat Forelimb Unloading Model to Understand Mechanical Influences on Postnatal Shoulder Development. 2018 NCUR 0(0) [cited 2018 Nov 8] Available from: http://www.ncurproceedings.org/ojs/index.php/NCUR2018/article/view/2673.

39. Crouch DL, Hutchinson ID, Plate JF, et al. 2015. Biomechanical Basis of Shoulder Osseous Deformity and Contracture in a Rat Model of Brachial Plexus Birth Palsy. J Bone Joint Surg Am 97(15):1264-1271.

40. Ward SR, Sarver JJ, Eng CM, et al. 2010. Plasticity of Muscle Architecture After Acute Supraspinatus Tear. J Orthop Sports Phys Ther 40(11):729-735.

41. Toursel T, Stevens L, Granzier H, Mounier Y. 2002. Passive tension of rat skeletal soleus muscle fibers: effects of unloading conditions. Journal of Applied Physiology 92(4):14651472.

42. Booth CM, Cortina-Borja MJ, Theologis TN. 2001. Collagen accumulation in muscles of children with cerebral palsy and correlation with severity of spasticity. Dev Med Child Neurol 43(5):314-320. 
477 43. Nikolaou S, Liangjun H, Tuttle LJ, et al. 2014. Contribution of denervated muscle to

478

479

480

481

482

483

484

485

486

487

488

489

490

491

492

493

494

495

496

497

498

499 contractures after neonatal brachial plexus injury: not just muscle fibrosis. Muscle Nerve 49(3):398-404.

44. Adams GR, Haddad F, Bodell PW, Tran PD, Baldwin KM. 2007. Combined isometric, concentric, and eccentric resistance exercise prevents unloading-induced muscle atrophy in rats. J Appl Physiol 103, 1644-54.

45. Hurst JE, Fitts RH. 2003. Hindlimb unloading-induced muscle atrophy and loss of function: protective effect of isometric exercise. J Appl Physiol 95: 1405-1417.

46. Ohira Y, Tanaka T, Yoshinaga T, Kawano F, Nomura T, Nonaka I, Allen DL, Roy RR, Edgerton VR. 2001. Ontogenetic, gravity-dependent development of rat soleus muscle. Am J Physiol Cell Physiol 280(4): C1008-16.

47. Brown M, Fisher JS, Salsich G. 1999. Stiffness and muscle function with age and reduced muscle use. J. Orthop. Res. 17, 409-414.

48. Deschenes MR, Adan MA, Kapral MC, Kressin KA, Leathrum CM, Seo A, Li S, Schaffrey EC. 2018. Neuromuscular Adaptability of Male and Female Rats to Muscle Unloading. J Neurosci Res 96(2): 284-96.

49. Zajac FE. Muscle and tendon: Properties, models, scaling, and application to biomechanics 303 and motor control. Crit Rev Biomed Eng. 1989;17(4):359.

50. Dixit NN, McCormick C, Warren E, Cole JH, Saul K (2019) Preganglionic and postganglionic brachial plexus injury effects on shoulder muscle growth. J Hand Surg .

51. Hultgren T, Einarsson F, Runesson E, Hemlin C, Friden J, Ljung BO. 2010. Structural characteristics of the subscapularis muscle in children with medial rotation contracture of the shoulder after obstetric brachial plexus injury. J Hand Surg Eur 35(1): 23-28. 
500 52. Gutmann E (ed.). 1962. The Denervated Muscle.

501 53. Goldspink DF. 1976. The Effects of Denervation on Protein Turnover of Rat Skeletal Muscle.

502 Biochem J 156, 71-80.

503 54. Goldspink DF. 1977. The influence of immobilization and stretch on protein turnover of rat 504 skeletal muscle. J Physiol 264, 267-282.

505 55. Kasper CE, Xun L. 2000. Expression of Titin in Skeletal Muscle Varies with Hind-Limb 506 Unloading. Biological Research for Nursing 2(2): 107-115. 


\section{$507 \quad$ Figures}

508

A)

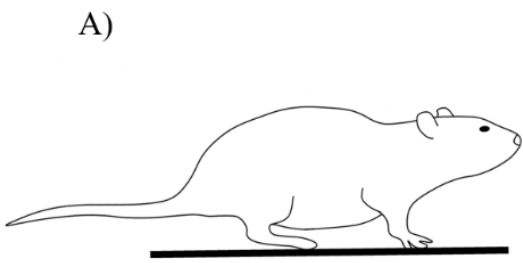

Control

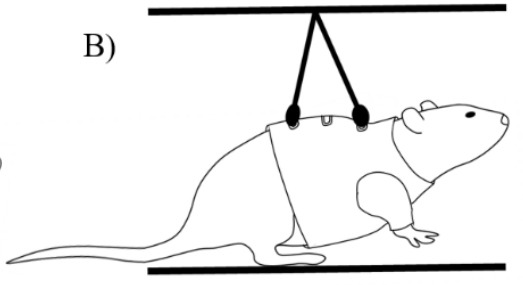

Forelimb Suspension

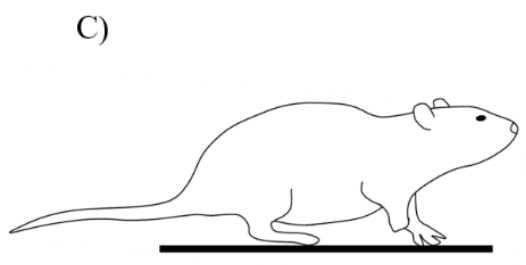

Forearm Amputation

510 Figure 1. The study design included a A) control group (both forelimbs unaffected by unloading;

511 right forelimb examined) and two unloading paradigms, B) forelimb suspension (both forelimbs

512 affected) and C) forearm amputation (right forelimb affected, left forelimb unaffected). 


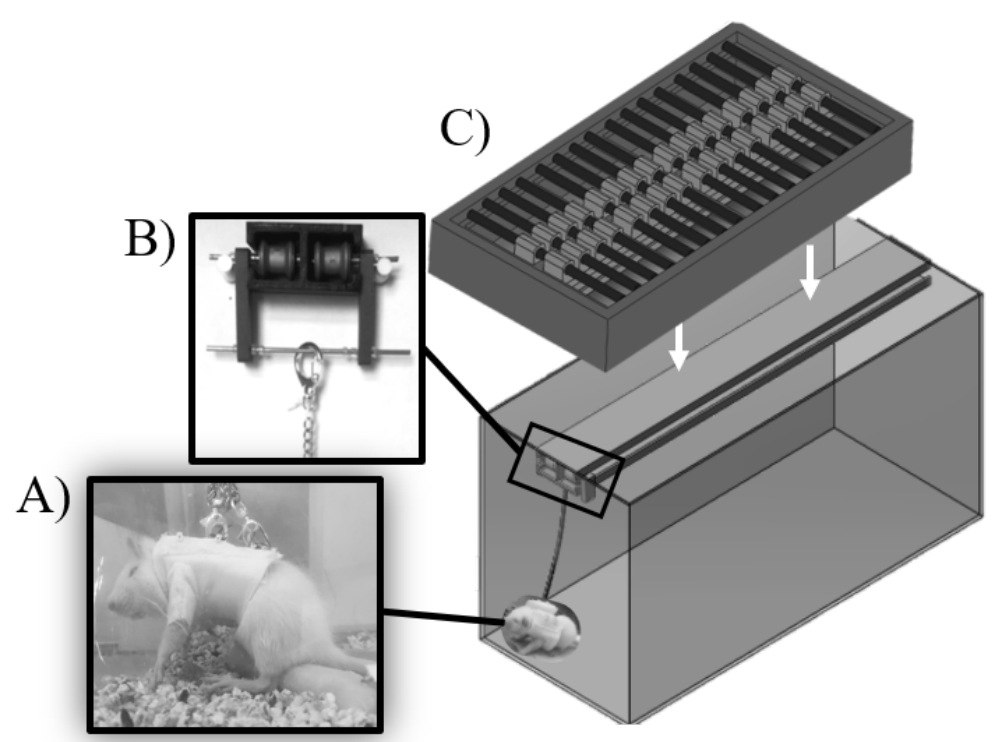

514 Figure 2. Custom forelimb suspension apparatus. A) Commercial harness sized to the growing rat

515 was tethered at two points using swivel hooks and adjustable chain and attached to the B) 3D

516 printed I-beam track system with low-friction wheels. C) Sixteen wooden dowels were inserted

517 into loops printed atop the track system to secure it within the lid. 


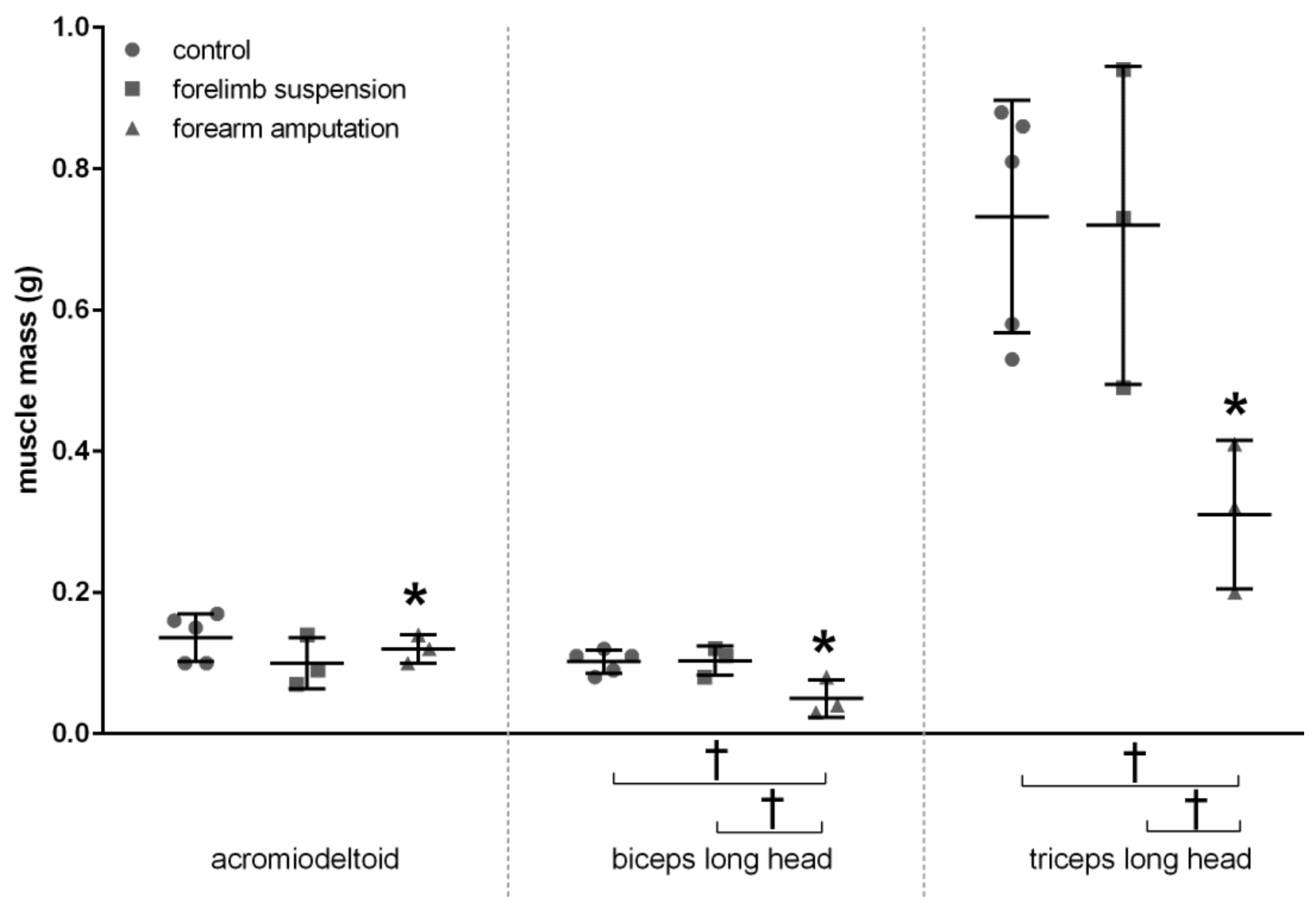

519 Figure 3. Muscle mass of forelimb muscles showing significant side-to-side differences (forearm

520 amputation only) and group differences (control, forelimb suspension, forearm amputation). Mean

$521 \pm$ standard deviation. $* \mathrm{p}<0.05$ for right vs. left limb. $\# \mathrm{p}<0.1$ (trend) for right vs. left limb. $\dagger \mathrm{p}<$ 5220.05 for group comparisons. 


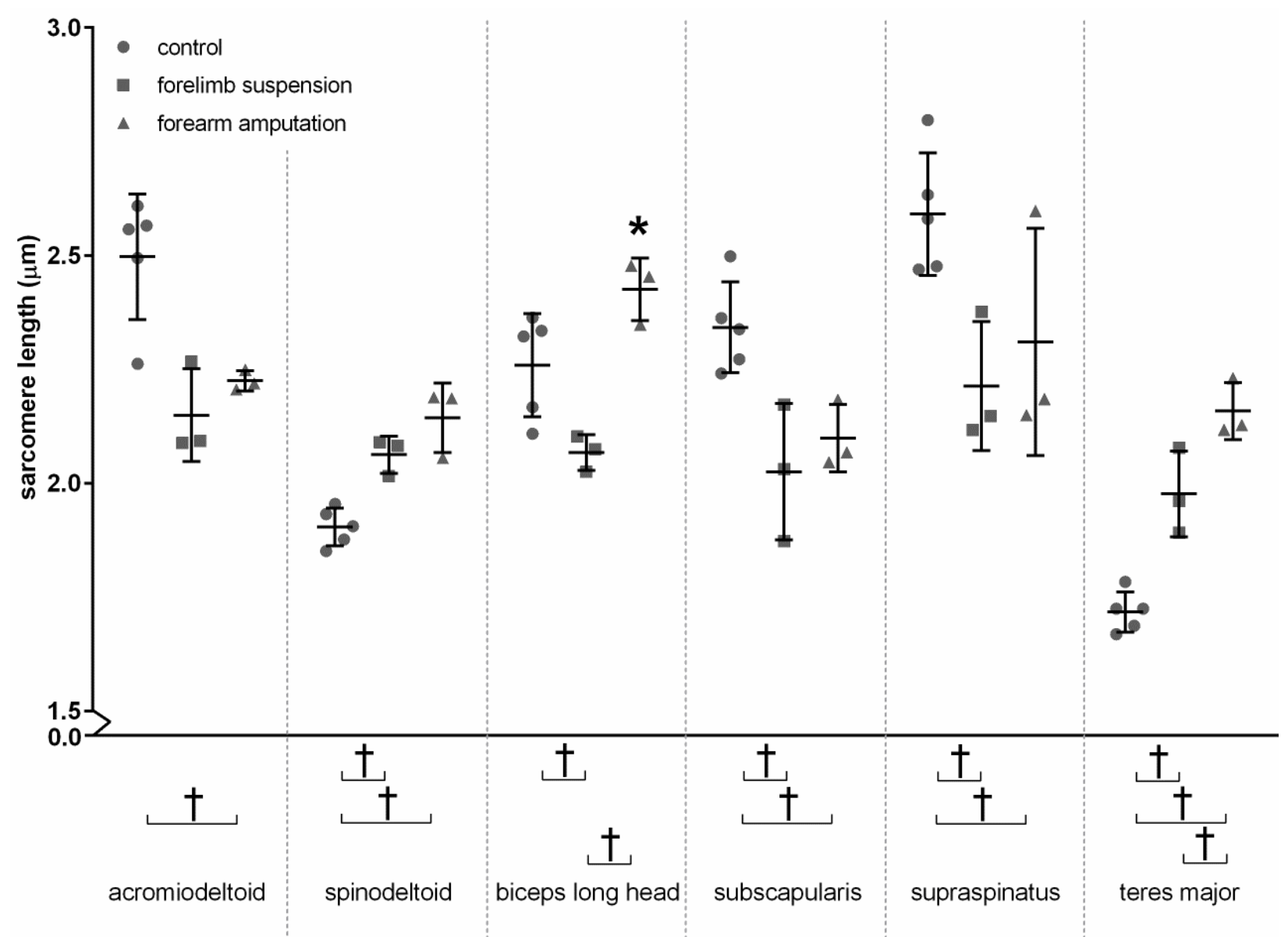

525 Figure 4. Muscle sarcomere lengths showing significant side-to-side differences (forearm 526 amputation only) and group differences (control, forelimb suspension, forearm amputation). Mean $527 \pm$ standard deviation. ${ }^{*} \mathrm{p}<0.05$ for right vs. left limb. $\# \mathrm{p}<0.1$ (trend) for right vs. left limb. $\dagger \mathrm{p}<$ 0.05 for group comparisons. $\$ \mathrm{p}<0.1$ (trend) for group comparisons. 


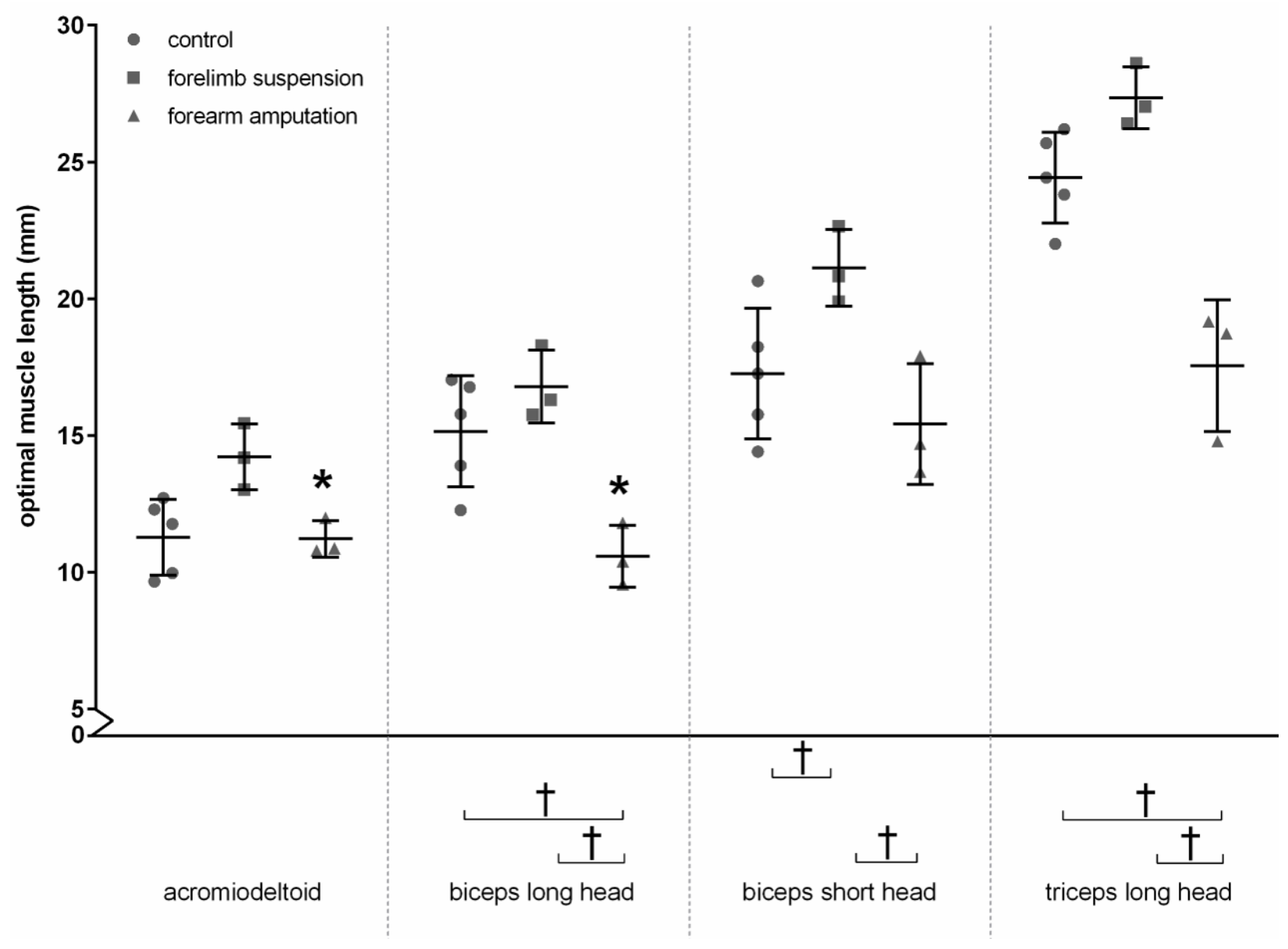

530 Figure 5. Optimal muscle lengths showing significant side-to-side differences (forearm

531 amputation only) and group differences (control, forelimb suspension, forearm amputation). Mean

$532 \pm$ standard deviation. ${ }^{*} \mathrm{p}<0.05$ for right vs. left limb. $\# \mathrm{p}<0.1$ (trend) for right vs. left limb. $\dagger \mathrm{p}<$

5330.05 for group comparisons. $\$ \mathrm{p}<0.1$ (trend) for group comparisons. 

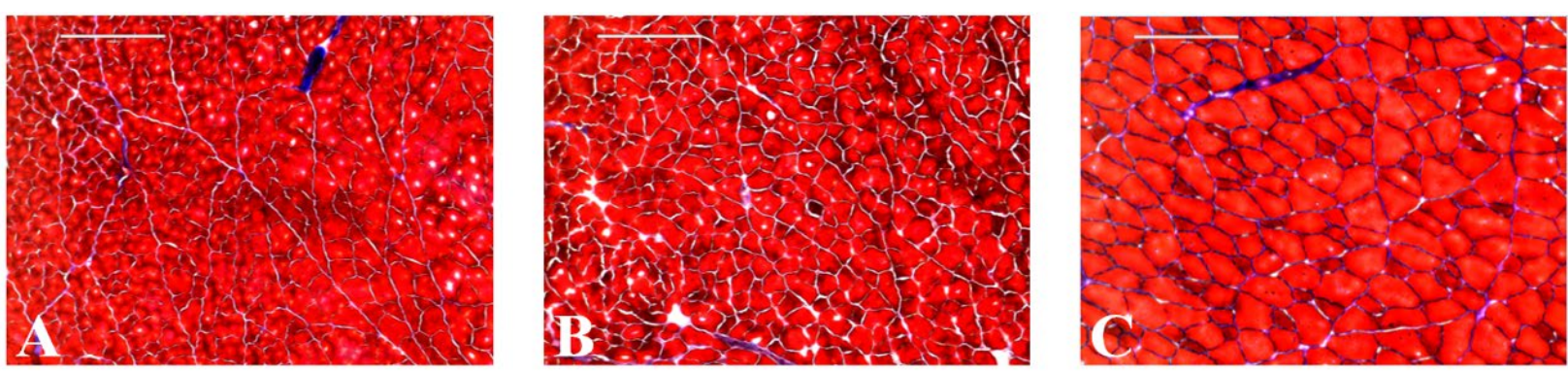

536 Figure 6. Longitudinal section of biceps long head muscle in A) control, B) forelimb suspension,

537 and C) forearm amputation groups, stained to assess collagen area (blue) as a percentage of muscle

538 tissue area (red). Scale bars $=200 \mu \mathrm{m}$. 
Tables

Table 1. Muscle mass, sarcomere length, and optimal muscle length for the control (left forelimb), standard deviation.

\begin{tabular}{lccc|ccc} 
& \multicolumn{3}{c}{$\begin{array}{c}\text { control } \\
\text { left (unaffected) }\end{array}$} & \multicolumn{3}{c}{$\begin{array}{c}\text { forelimb suspension } \\
\text { right (affected) }\end{array}$} \\
\cline { 2 - 7 } & $\begin{array}{c}\text { mass } \\
\text { sarcomere length }\end{array}$ & $\begin{array}{c}(\mu \text { optimal length } \\
(\mathrm{g})\end{array}$ & $\begin{array}{c}\text { mass } \\
(\mathrm{g})\end{array}$ & $\begin{array}{c}\text { sarcomere length } \\
(\mu \mathrm{m})\end{array}$ & $\begin{array}{c}\text { optimal length } \\
(\mathrm{mm})\end{array}$ \\
\cline { 2 - 7 } pectoralis major & $0.34 \pm 0.08^{\mathrm{a}}$ & $2.33 \pm 0.27$ & $19.4 \pm 2.1$ & $0.23 \pm 0.03$ & $1.92 \pm 0.08$ & $22.2 \pm 3.3$ \\
acromiodeltoid & $0.14 \pm 0.03$ & $2.49 \pm 0.14$ & $11.3 \pm 1.4$ & $0.10 \pm 0.03$ & $2.15 \pm 0.10$ & $14.2 \pm 1.2$ \\
spinodeltoid & $0.14 \pm 0.03$ & $1.90 \pm 0.04$ & $20.1 \pm 1.8$ & $0.12 \pm 0.05$ & $2.06 \pm 0.04$ & $25.0 \pm 2.4$ \\
biceps long head & $0.10 \pm 0.02$ & $2.26 \pm 0.11$ & $15.2 \pm 2.0$ & $0.10 \pm 0.02$ & $2.07 \pm 0.04$ & $16.8 \pm 1.3$ \\
biceps short head & $0.01 \pm 0.01$ & $2.09 \pm 0.21$ & $17.3 \pm 2.4$ & $0.04 \pm 0.02$ & $1.95 \pm 0.06$ & $21.1 \pm 1.4$ \\
subscapularis & $0.27 \pm 0.05$ & $2.34 \pm 0.10$ & $18.2 \pm 3.0$ & $0.19 \pm 0.06$ & $2.03 \pm 0.15$ & $21.6 \pm 0.6$ \\
supraspinatus & $0.21 \pm 0.06$ & $2.59 \pm 0.13$ & $20.2 \pm 2.2$ & $0.20 \pm 0.05$ & $2.21 \pm 0.14$ & $23.7 \pm 1.0$ \\
infraspinatus & $0.22 \pm 0.05$ & $2.37 \pm 0.10$ & $22.1 \pm 2.6$ & $0.19 \pm 0.02$ & $2.22 \pm 0.05$ & $24.0 \pm 2.0$ \\
teres major & $0.21 \pm 0.05$ & $1.72 \pm 0.04$ & $24.5 \pm 2.8$ & $0.21 \pm 0.05$ & $1.98 \pm 0.09$ & $25.0 \pm 0.9$ \\
triceps long head & $0.73 \pm 0.16$ & $1.98 \pm 0.13$ & $24.4 \pm 1.7$ & $0.72 \pm 0.23$ & $1.89 \pm 0.03$ & $27.3 \pm 1.1$
\end{tabular}

${ }^{a}$ Pectoralis major muscle mass was lower in right vs. left limb, likely due to sham surgery in previous study.

\section{forearm amputation}

left (unaffected)

right (affected)

\begin{tabular}{ccc|ccc}
\hline $\begin{array}{c}\text { mass } \\
(\mathrm{g})\end{array}$ & $\begin{array}{c}\text { sarcomere length } \\
(\mu \mathrm{m})\end{array}$ & $\begin{array}{c}\text { optimal length } \\
(\mathrm{mm})\end{array}$ & \multicolumn{1}{c}{$\begin{array}{c}\text { mass } \\
(\mathrm{g})\end{array}$} & $\begin{array}{c}\text { sarcomere length } \\
(\mu \mathrm{m})\end{array}$ & $\begin{array}{c}\text { optimal length } \\
(\mathrm{mm})\end{array}$ \\
\hline $0.27 \pm 0.15$ & $2.07 \pm 0.08$ & $31.5 \pm 10.1$ & $0.26 \pm 0.07$ & $2.21 \pm 0.07$ & $23.4 \pm 2.5$ \\
$0.15 \pm 0.03$ & $2.18 \pm 0.03$ & $15.4 \pm 0.8$ & $0.12 \pm 0.02^{*}$ & $2.23 \pm 0.02$ & $11.2 \pm 0.7^{*}$ \\
$0.13 \pm 0.03$ & $2.19 \pm 0.01$ & $23.7 \pm 2.7$ & $0.13 \pm 0.01$ & $2.14 \pm 0.08$ & $16.8 \pm 6.9$ \\
$0.11 \pm 0.04$ & $2.06 \pm 0.08^{*}$ & $17.5 \pm 1.0$ & $0.05 \pm 0.03^{*}$ & $2.43 \pm 0.07$ & $10.6 \pm 1.1^{*}$ \\
$0.02 \pm 0.01$ & $1.99 \pm 0.01$ & $19.1 \pm 0.2$ & $0.03 \pm 0.01$ & $2.22 \pm 0.13$ & $15.4 \pm 2.2$ \\
$0.20 \pm 0.10$ & $2.14 \pm 0.09$ & $23.9 \pm 2.8$ & $0.28 \pm 0.06$ & $2.10 \pm 0.07$ & $22.3 \pm 0.9$ \\
$0.24 \pm 0.11$ & $2.33 \pm 0.11$ & $24.2 \pm 1.9$ & $0.14 \pm 0.05$ & $2.31 \pm 0.25$ & $21.6 \pm 3.5$ \\
$0.22 \pm 0.03$ & $2.37 \pm 0.13$ & $25.0 \pm 2.8$ & $0.17 \pm 0.03$ & $2.30 \pm 0.05$ & $21.1 \pm 1.7$ \\
$0.23 \pm 0.04$ & $2.05 \pm 0.05$ & $24.9 \pm 1.3$ & $0.22 \pm 0.06$ & $2.16 \pm 0.06$ & $22.6 \pm 0.7$ \\
$0.71 \pm 0.18$ & $2.03 \pm 0.05$ & $24.6 \pm 2.7$ & $0.31 \pm 0.11^{*}$ & $2.07 \pm 0.10$ & $17.6 \pm 2.4$
\end{tabular}

${ }^{*} \mathrm{p}<0.05$ for smaller value vs. contralateral limb. 
546 Table 2. Percent collagen content for the control (left forelimb), forelimb suspension (right

547 forelimb), and forearm amputation (right forelimb) groups. Mean \pm standard deviation.

\begin{tabular}{lccc} 
& control & forelimb suspension & forearm amputation \\
\cline { 2 - 4 } biceps long head & $5.9 \pm 1.3$ & $5.9 \pm 0.8^{\mathrm{a}}$ & $5.9 \pm 0.0^{\mathrm{b}}$ \\
biceps short head & $6.0 \pm 1.3$ & $4.7 \pm 0.3^{\mathrm{a}}$ & $7.9 \pm 0.0^{\mathrm{b}}$ \\
upper subscapularis & $5.8 \pm 2.0$ & $4.1 \pm 0.5$ & $5.5 \pm 1.7$ \\
lower subscapularis & $8.1 \pm 2.0^{\mathrm{a}}$ & $4.3 \pm 1.1$ & $4.4 \pm 1.0$
\end{tabular}

${ }^{\text {aNot }}$ all specimens could to be imaged.

${ }^{\mathrm{b}}$ Not all specimens could be sectioned. 